\title{
Fenquizone Potassium
}

National Cancer Institute

\section{Source}

National Cancer Institute. Fenquizone Potassium. NCI Thesaurus. Code C95125.

The potassium salt form of fenquizone, a quinazolinone structurally related to metolazone, and sulphonamide-based diuretic with diuretic activity. As a thiazide-like diuretic, fenquizone acts by blocking reabsorption of sodium in the proximal tubule and the ascending branch of the loop of Henle. This causes an increase in diuresis with the elimination of chloride and sodium. 